\title{
Continuous-wave operation of electrically pumped, single-mode, edge-emitting photonic crystal Bragg lasers
}

\author{
Lin Zhu, ${ }^{\text {a) }}$ Xiankai Sun, Guy A. DeRose, Axel Scherer, and Amnon Yariv \\ Department of Electrical Engineering, California Institute of Technology, \\ 1200 E. California Blvd., Pasadena, California 91125 and Department of Applied Physics, California \\ Institute of Technology, 1200 E. California Blvd., Pasadena, California 91125
}

(Received 13 March 2007; accepted 3 June 2007; published online 28 June 2007)

\begin{abstract}
The authors demonstrate an electrically pumped, single-mode, large-area, edge-emitting InGaAsP/InP two dimensional photonic crystal Bragg laser operating in continuous-wave condition. The laser uses a weak index perturbed, polymer-planarized, surface photonic crystal structure to control the optical mode in the wafer plane. They find that the laser operates in single transverse and longitudinal modes. They compare the performance of the photonic crystal Bragg laser with a broad-area laser fabricated from the same wafer and the comparison shows that the performance penalty incurred by the photonic crystal is small. (C) 2007 American Institute of Physics. [DOI: 10.1063/1.2752124]
\end{abstract}

Two dimensional photonic crystal (PC) structures with a weak periodic refractive index perturbation can provide the necessary modal control in the wafer plane for a wide aperture laser. These structures have been engineered to make large-modal-volume, high-power semiconductor lasers with narrow spectral linewidths and narrow beam divergences. ${ }^{1-7}$ We have recently reported electrically pumped, large-area, edge-emitting photonic crystal Bragg lasers operating in pulsed condition. ${ }^{7}$ Furthermore, we demonstrated that we could systematically tune the lasing wavelength by changing the PC lattice constants, thus demonstrating that the lasing mode is truly defined by both the longitudinal and transverse Bragg conditions. However, due to the pulsed excitation and lack of temperature control, the lasing spectra were obtained under transient conditions with the attendant broadening. It was difficult for us to draw conclusions about single or multimodedness.

In this letter, we report continuous-wave $(\mathrm{CW})$ electrically pumped, single-mode InGaAsP/InP PC Bragg lasers with angled facets operating in a temperature range of 140-180 K. We measure the output light power verse pump current $(L-I)$ curves for the PC laser at different temperatures. We compare the laser threshold current and slope efficiency with those of a straight-facet broad-area (BA) laser fabricated from the same wafer with the same size and under the same operating conditions. We show that the performance penalty in terms of threshold current and slope efficiency incurred by the presence of lasing in a photonic crystal mode is small. This is important for practical applications of our PC lasers. We measure the PC laser linewidth with an optical heterodyne approach ${ }^{8}$ and show that the laser truly operates in a single mode. We also measure the laser spectra at different temperatures and obtain a temperature tuning sensitivity $d \lambda / d T$ of $0.09 \mathrm{~nm} /{ }^{\circ} \mathrm{C}$, which is similar to a regular distributed feedback (DFB) laser. ${ }^{9}$

Figure 1 shows a schematic diagram of our PC Bragg laser. The photonic crystal consists of a rectangular lattice array of polymer-filled holes each with a radius of $100 \mathrm{~nm}$ on the wafer surface. The transverse and longitudinal lattice

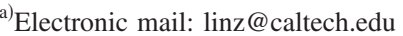

constants are $a$ and $b$, respectively. The cleaved facet is titled relative to the transverse lattice direction by an angle $\theta_{\text {tilt }}$. Angled facets are used to suppress effective index-guided modes and gain-guided modes and to ensure the lasing of Bragg-guided modes only. ${ }^{10-12}$

The wafer structure and fabrication procedure are detailed in Refs. 6 and 7. The PC pattern is first written on the wafer surface by electron beam lithography and is then etched into the semiconductor about $380 \mathrm{~nm}$ to obtain the desired index contrast. The polyimide planarization is then realized by an etch-back process and creates a polyimide post inside each etched hole. The polyimide separates the etched holes from the subsequently evaporated metal contact to reduce the optical loss and help obtain good contact quality.

We use a first order Bragg reflection for the transverse direction with a lattice constant of $a=1 \mu \mathrm{m}$ and a second order Bragg reflection for the longitudinal direction with a lattice constant of $b=470 \mathrm{~nm}$. The design corresponds to a resonance wavelength of $1483 \mathrm{~nm}$ (the effective index $n_{\mathrm{eff}}$ is estimated to be 3.24). The metal contact width is about $100 \mu \mathrm{m}$ and the facet tilt angle is $13.2^{\circ}$ (the transverse resonance angle of our design, see Ref. 7). Fabricated lasers are cleaved to lengths of about $450 \mu \mathrm{m}$ and widths of about $400 \mu \mathrm{m}$. Each cleaved laser is then bonded to a $C$-mount and placed in a microcryogenic refrigerator (MMR Technology).

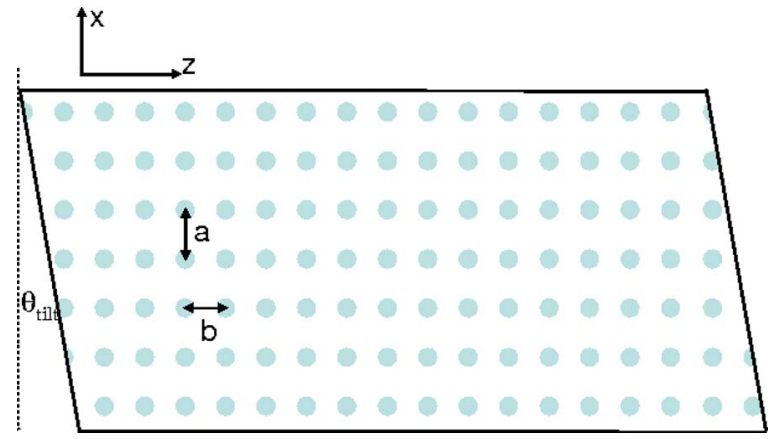

FIG. 1. (Color online) Schematic of a two dimensional photonic crystal Bragg laser. $a$ is the transverse lattice constant, $b$ is the longitudinal lattice constant, and $\theta_{\text {tilt }}$ is the facet tilt angle. 

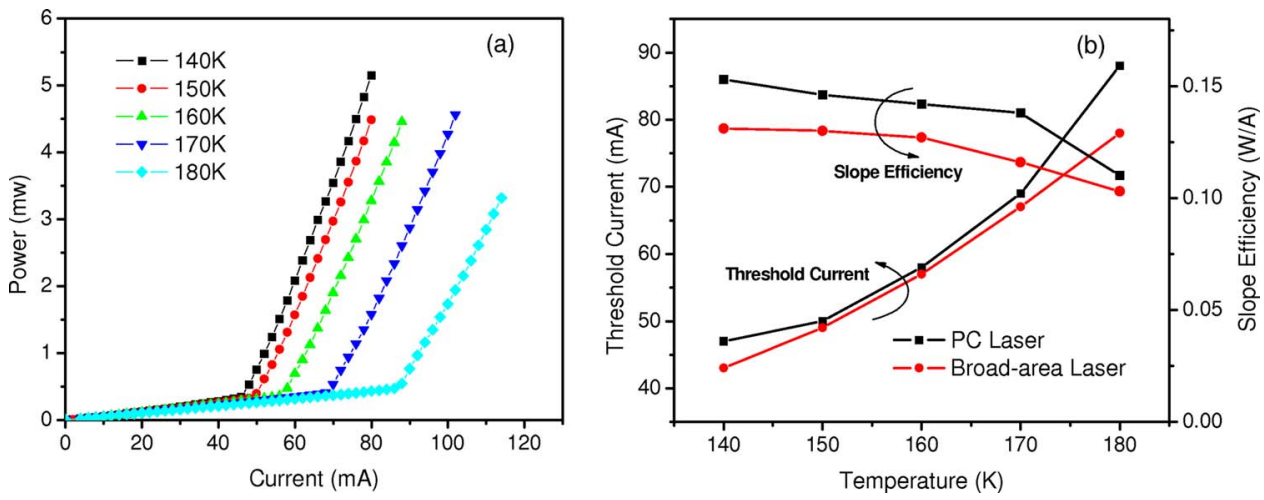

FIG. 2. (Color online) (a) $L-I$ curves for the photonic crystal Bragg laser at different temperatures. (b) Comparison of the threshold current and slope efficiency between the photonic crystal Bragg laser and broad-area laser.

We test the lasers in the temperature range of $140-180 \mathrm{~K}$ to obtain the $\mathrm{CW}$ lasing.

The PC Bragg laser $L-I$ curves at different temperatures are shown in Fig. 2(a). In the test temperature range, the threshold current varies from 47 to $88 \mathrm{~mA}$. We optimize the measurement setup and make sure that the output light collection efficiency is the same for different tests. Thus we can compare the threshold current and slope efficiency between the PC Bragg laser and the BA laser. The comparison results are shown in Fig. 2(b).

The PC Bragg laser is theoretically expected to have a lower threshold due to the gain enhancement at the band edge. However, the PC structure fabricated by a dry-etching technique causes extra losses due to the scattering and surface recombination, which leads to a higher threshold. In Fig. 2(b), the threshold current for the PC Bragg laser is slightly higher than the BA laser. As the temperature increases, the material gain decreases and gain peak shifts to longer wavelengths. The threshold of the BA laser is determined by the material peak gain and varies almost linearly with the temperature in our experiment. In contrast, the threshold of the PC Bragg laser is determined by the gain at the resonance wavelength. In Fig. 2(b), the PC Bragg laser exhibits higher slope efficiency than the BA laser despite the extra losses imposed by the PC structure. This is mainly due to the fact that the PC structure leads to a wide optical mode and prevents the formation of filamentation. The comparison results show that our PC Bragg lasers can perform similarly to or better than the BA laser fabricated from the material in terms of the threshold current and slope efficiency, and these PC Bragg lasers are useful for practical applications.

Figure 3(a) shows the lasing spectrum at the pump current $I=1.3 I_{\text {th }}$ and the temperature $T=150 \mathrm{~K}$. The side mode suppression ratio is about $32 \mathrm{~dB}$. The full width at half maximum ( $3 \mathrm{~dB}$ linewidth) is $0.15 \mathrm{~nm}$, which is limited by the resolution of our optical spectrum analyzer $(0.08 \mathrm{~nm})$. In order to resolve the fine features of the lasing spectrum, we use an optical heterodyne detection approach. We couple the light from the PC laser into a single mode fiber and combine it with the light from a tunable laser using a $3 \mathrm{~dB}$ fiber coupler. The optical beating signal is then photodetected by a $25 \mathrm{GHz}$ photodiode. The spectrum of the electrical current is then measured on a $50 \mathrm{GHz}$ electrical spectrum analyzer. We set the wavelength of the tunable laser output light the same as the PC Bragg laser and sweep the wavelength in a range of $0.2 \mathrm{~nm}$ with a step of $0.001 \mathrm{~nm}$. A single-peaked electrical spectrum is observed and is shown in Fig. 3(b). This shows that the PC Bragg laser operates in a single mode and the linewidth of the PC laser is estimated to be $110 \mathrm{MHz}$ from Fig. 3(b). This linewidth is broader compared to a commercial DFB laser and it is possibly due to the material, metal contact, and temperature nonuniformity across a large lasing area $\left(100 \times 450 \mu \mathrm{m}^{2}\right)$.

It should be pointed out that the laser operates at the band edge of the photonic crystal. Although the photonic crystal possesses a fourfold rotational symmetry, the laser operates in a single mode due to additional perturbing reflections from the cleaved facets. This additional perturbation breaks the unwanted degeneracy as in regular DFB lasers with cleaved facets. ${ }^{13}$

Figure 4(a) shows the lasing spectra at the pump current $I=1.6 I_{\text {th }}$ when the temperature changes from 140 to $170 \mathrm{~K}$. The side mode suppression ratios of the spectra at different temperatures are all greater than $30 \mathrm{~dB}$. The lasing wavelength changes from 1483.95 to $1486.80 \mathrm{~nm}$ as the temperature changes from 140 to $170 \mathrm{~K}$. This corresponds to a temperature tuning sensitivity $d \lambda / d T$ of $0.09 \mathrm{~nm} /{ }^{\circ} \mathrm{C}$ and it is similar to a regular DFB laser. ${ }^{9}$ The wavelength change is mainly due to the refractive index change with the tempera-
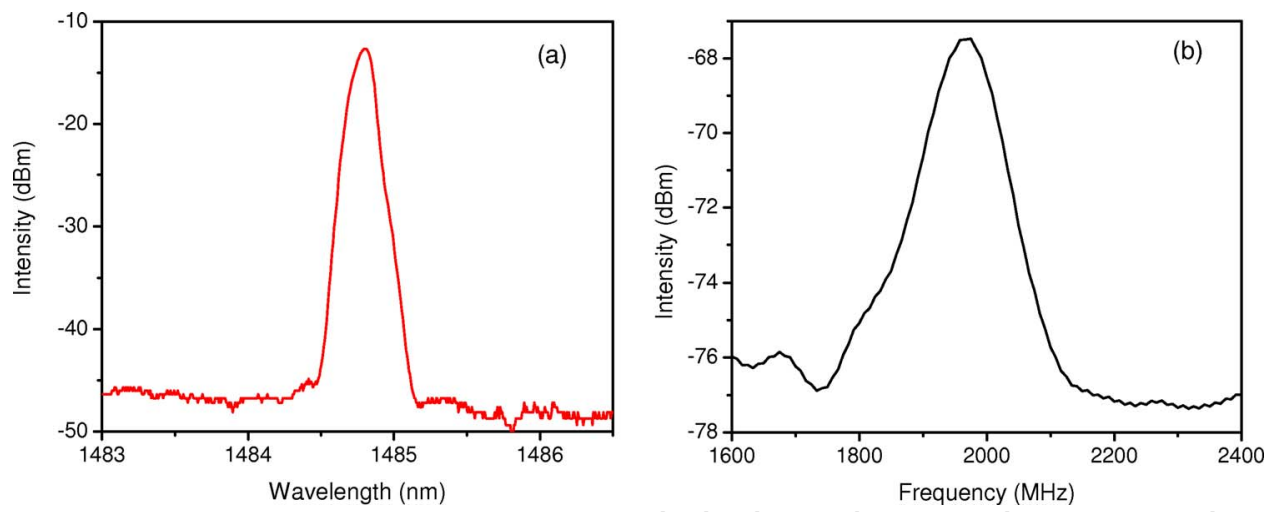

FIG. 3. (Color online) (a) Lasing spectrum of the PC Bragg laser at $1.3 I_{\text {th }}$ $(150 \mathrm{~K})$. (b) Electrical spectrum of the beating signal between the PC Bragg laser and a tunable laser. 

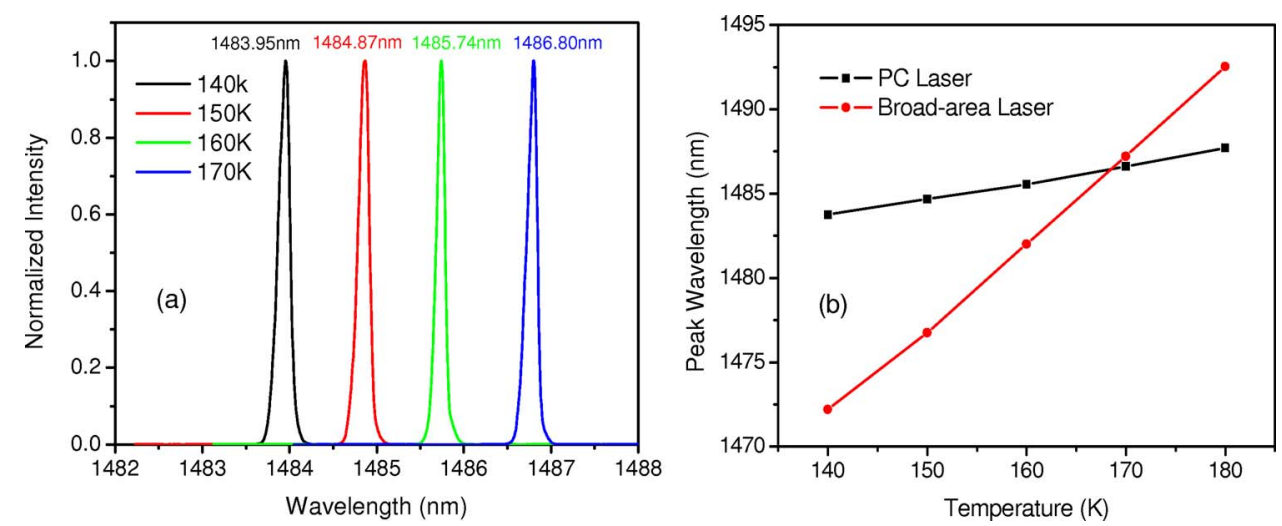

FIG. 4. (Color online) (a) Lasing spectra of the PC laser at different temperatures $\left(1.6 I_{\mathrm{th}}\right)$. (b) Laser peak wavelength as a function of the temperature for the $\mathrm{PC}$ laser and BA laser. ture. Figure 4(b) compares the temperature tuning sensitivity for the lasing wavelength between the PC laser and BA laser. For the BA laser, the temperature tuning sensitivity is about $0.5 \mathrm{~nm} /{ }^{\circ} \mathrm{C}$, which is similar to the temperature tuning sensitivity of the material gain spectrum peak. ${ }^{9}$

In summary, we have demonstrated electrically pumped, large-area, edge-emitting photonic crystal lasers in InGaAsP active semiconductor materials operating under CW condition. Single-mode lasing is obtained for $100 \mu \mathrm{m}$ wide and $450 \mu \mathrm{m}$ long devices at a temperature range of 140-180 K. We compare the threshold and slope efficiency of the PC laser with the BA laser and show that performance penalty incurred by the imposition of lasing in a photonic crystal mode is small. These results constitute a sizable step on the way to broad-area, single-mode, high-power semiconductor lasers and are important for implementing our photonic crystal lasers in practical applications.

One of the authors (L.Z) would like to thank O. Painter and R. Perahia for providing access to their plasma-enhanced chemical-vapor deposition facility. P. Chak, J. Choi, J. Poon, and W. Liang are acknowledged for fruitful discussions. This work was supported by the Airforce Office of Scientific Re- search (H. Schlossberg) and by the DARPA (J. Mangano).

${ }^{1}$ I. Vurgaftman and J. R. Meyer, IEEE J. Quantum Electron. 38, 592 (2002).

${ }^{2}$ C. S. Kim, W. W. Bewley, C. L. Canedy, I. Vurgaftman, M. Kim, and J. R. Meyer, IEEE Photonics Technol. Lett. 16, 1250 (2004).

${ }^{3}$ S. Noda, M. Yokoyama, M. Imada, A. Chutinan, and M. Mochizuki, Science 293, 1123 (2001)

${ }^{4}$ D. Ohnishi, T. Okano, M. Imada, and S. Noda, Opt. Express 12, 1562 (2004).

${ }^{5}$ L. Zhu, J. M. Choi, G. A. DeRose, A. Yariv, and A. Scherer, Opt. Lett. 31, 1863 (2006)

${ }^{6}$ L. Zhu, P. Chak, J. K. S. Poon, G. A. Derose, A. Yariv, and A. Scherer, Opt. Express 15, 5966 (2007).

${ }^{7}$ L. Zhu, G. A. DeRose, A. Scherer, and A. Yariv, Opt. Lett. 32, 1256 (2007).

${ }^{8}$ D. Derickson, Fiber Optic Test and Measurement (Prentice-Hall, Englewood Cliffs, NJ 1998), pp. 283-285.

${ }^{9}$ G. P. Agrawal and N. K. Dutta, Long-Wavelength Semiconductor Lasers (Van Nostrand Reinhold, New York, 1986), pp. 314-316.

${ }^{10}$ R. J. Lang, K. D. Zurko, A. Hardy, S. Demars, A. Schoenfelder, and D. Welch, IEEE J. Quantum Electron. 34, 2196 (1998).

${ }^{11}$ A. M. Sarangan, M. W. Wright, J. Marciante, and D. J. Bossert, IEEE J. Quantum Electron. 35, 1220 (1999).

${ }^{12}$ L. Zhu, A. Yariv, and A. Scherer (unpublished).

${ }^{13}$ L. A. Coldren and S. W. Corzine, Diode Lasers and Photonic Integrated Circuits (Wiley Interscience, New York, 1995), pp. 103-105. 